\title{
Reduction of Transmitted Information Using Similarities between Range Blocks in Fractal Image Coding
}

\author{
Xiaotong $\mathrm{Hu}^{*}$ Shuping Qiu* and Hideo Kuroda ** \\ * Tianjin University of Science and Technology, \\ 1038 Dagu Nanlu, Hexi District, Tianjin, P.R.china \\ ** Nagasaki University, Nagasaki, Japan \\ E-mail:huxt@tust.edu.cn
}

\begin{abstract}
Fractal image coding uses the similarities between the best matching domain blocks and range blocks to reconstruct image. In the transmitted information, the information about the best matching domain blocks occupies a large percentage, so the reduction of information about the best matching domain blocks is the most effective method to reduce the quality of transmitted information. On the other hand, there are similarities between range blocks from each other. So, when range blocks are similar enough, we can reconstruct a range block by its similar range block, in other words, we can use the same domain block as their best matching domain block between similar range blocks. In this paper, we propose a method using similarities between the range blocks to reduce the position and affine information of the best matching domain blocks by sharing the same best matching domain block between similar range blocks.
\end{abstract}

\section{Introduction}

The fractal image coding was advocated by Barnsley in 1988, Jacquin who was a student of Barnsley performed a concrete research of fractal image coding. As it uses self-transformability as one of the image characteristics, fractal image coding has been studied in recent years intensively [1]-[8].

In fractal image coding, range blocks are reconstructed by their best matching domain blocks selected by similarities between range blocks and domain blocks. Therefore, fractal image coding has always been regarded similarity between domain blocks and range blocks as importance. The encoder transfers position and affine information of the best matching domain blocks to the decoder to reconstruct images. In the transmitted information, the information about the best matching domain block shown above occupies a high percentage.
In order to reduce the transmitted information, much research has been carried out. Jacquin[1] defined three types of blocks; shade, midrange and edge. Shade blocks are flat, and the others are non-flat. If a range block belongs to the shade block type, it is approximated to the mean value without any searching of the best matching domain blocks. Range blocks defined as midrange and edge block types selected the best matching domain blocks in midrange and edge domain pools, respectively. Popescu[4] proposed a method to search for high priority domain blocks which have a moment similar to a given range block. As mentioned above, all current methods use only similarities between range and domain blocks.

On the other hand, as there exist similarities between range blocks and domain blocks, it is possible that the similarity also exists between range blocks. In this paper, we examine the similarity between range blocks, and propose a new method of reducing quantity of transmitted information using the similarity between range blocks.

Section 2 introduces similarities between range blocks; section 3 introduces reduction in transmitted information by similarities between range blocks; section 4 introduces algorithm of the proposed method; section 5 introduces transmitted information in the proposed method; section 6 introduces the results of simulations; section 7 is conclusion.

\section{Similarities between range blocks in fractal image coding}

\subsection{Fractal image coding}

In fractal image coding, an original image is subdivided into small blocks, which are called range blocks while the bigger image blocks are defined as

The Project-sponsored by SRF for ROCS, SEM. 
domain blocks. Range blocks are divided into flat or non-flat range blocks by their standard deviation. The domain block $d$ is determined as the best matching domain block of range block when its distance $\rho_{r d}$ from $r$ is shown in (1) as the smallest value.

$$
\rho_{r d}=\sqrt{\frac{1}{n^{2}} \times \sum_{i=0}^{n-1} \sum_{j=0}^{n-1}\{r(i, j)-d(i, j)\}^{2}}
$$

$n$ : size of range block and contracted domain block

$r(i, j)$ : pixel value at position $(i, j)$ of range block $r$

$d(i, j)$ : pixel value at position $(i, j)$ of domain block $d$

Then, the position and affine transformation information of the best matching domain blocks and mean values plus standard deviation of range block are sent to the decoder.

In the decoder, the low-resolution initial image is reconstructed only by the mean values for each range block, and save initial image in frame memory. Based on this initial image, iteration processes are performed as follows to reconstruct non-flat range blocks.

The domain blocks are extracted from the location of the best matching domain blocks, and then the extracted domain blocks are contracted to the range block size and the contracted blocks are oriented by affine information. And then we can use this domain block to reconstruct range block shown in (2).

$$
r_{i j}=\left(d_{i j}-M_{d}\right) \cdot \frac{S_{r}}{S_{d}}+M_{r}
$$

In (2), $r_{i j}$ is the pixel value of new range block. The value $S_{r}$ and $S_{d}$ are the standard deviations of the range block and domain blocks, $M_{r}$ is the received mean value of the range block, while $d_{i j}$ is the oriented and rescaled pixel value of domain block, and $M_{d}$ is the mean value of oriented and rescaled domain block.

This processing is repeated until the image of enough resolution is obtained or the change between the images settles.

\subsection{Similarities between range blocks}

In order to examine the similarities between range blocks, we can search for similar range blocks as well as the best matching domain block of range block is searched in the current method.
We set range block $R_{2}$ as the best matching range block of range block $R_{1}$ when $\delta_{R_{1} R_{2}}$ shown in (3) between $R_{1}$ and $R_{2}$ is smallest.

$$
\begin{aligned}
& \delta_{R_{1} R_{2}}=\left\{\frac{1}{n^{2}} \times \sum_{i=0}^{n-1} \sum_{j=0}^{n-1}\left[r_{1}(i, j)-r_{2}(i, j)\right]^{2}\right\}^{\frac{1}{2}} \\
& r_{1}(i, j): \text { Pixel value at position }(i, j) \text { of range } \\
& \text { block } R_{1} \\
& r_{2}(i, j): \text { Pixel value at position }(i, j) \text { of range } \\
& \text { block } R_{2}
\end{aligned}
$$

Euclidean distance $D$ between $R_{1}$ and the best matching range block $R_{2}$ is defined in (4).

$$
D=\sqrt{\left(x_{1}-x_{2}\right)^{2}+\left(y_{1}-y_{2}\right)^{2}}
$$

$\left(x_{1}, y_{1}\right)$ : Coordinates of pixel at the left-top of range block $R_{1}$

$\left(x_{2}, y_{2}\right)$ : Coordinates of pixel at the left-top of range block $R_{2}$

We examined frequencies and $D$ between range blocks most similar to each other, result is shown in Figure 1 when the test image Lenna is used. In Figure 1, $\mathrm{X}$-axis is the Euclidean distance between the best matching range blocks; the $\mathrm{Y}$-axis is the frequencies of the best matching range blocks.

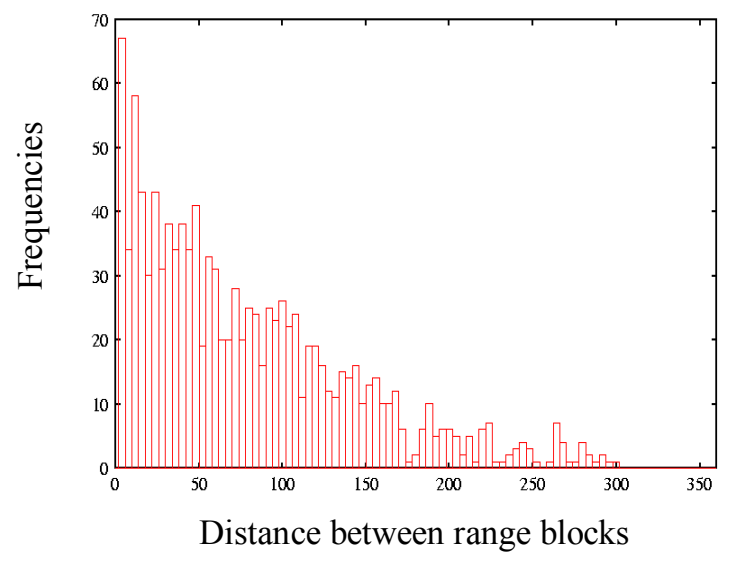

Figure 1. Frequencies and Euclidean distance between the best matching range blocks.

As shown in Figure 1, the smaller the Euclidean distance between range blocks is, the higher the frequencies of the best matching range block become. In particular, values smaller than 12 in Euclidean 
distance, the best matching range block occur quite often.

\section{Reduction in the transmitted information by similarities between range bocks}

In fractal image encoding processing, range blocks are divided into two kinds that is flat range block and non-flat range block with their standard deviations. And then, to flat range block, their mean values are transmitted only. On the other hand, it searches for the best matching domain blocks to the non-flat range blocks, and information shown in Table 1 is transmitted to the decoder.

As shown in Table 1, position and affine transformation information of the best matching domain block occupy a large percentage of transmitted information. Therefore, it is an effective method of reducing transmitted information to reduce information about the best matching domain blocks.

As an information reduction method, similar range blocks are reconstructed with the same best matching domain block as thought. Because the number of range blocks is fewer than that of domain blocks, so the address of range block is shorter than that of domain blocks. As a result, the method to share the best domain block between similar range blocks is considered as an information reduction method.

Table 1. Quantity of transmitted information in the current method

\begin{tabular}{|l|c|c|c|}
\hline \multicolumn{1}{|c|}{ Information name } & Sign & Lenna $\begin{array}{c}\text { Other } \\
\text { images }\end{array}$ \\
\hline Flat/non-flat flag & $F_{1}$ & \multicolumn{2}{|c|}{$1[\mathrm{bit}]$} \\
\hline $\begin{array}{l}\text { Mean value of the range } \\
\text { block }\end{array}$ & $M_{r}$ & \multicolumn{2}{|c|}{$8[\mathrm{bits}]$} \\
\hline $\begin{array}{l}\text { Affine information of } \\
\text { the best matching } \\
\text { domain block }\end{array}$ & $\tau$ & \multicolumn{2}{|c|}{$3[\mathrm{bits}]$} \\
\hline $\begin{array}{l}\text { Standard deviation of } \\
\text { the range block }\end{array}$ & $S_{r}$ & \multicolumn{2}{|c|}{$7[\mathrm{bits}]$} \\
\hline $\begin{array}{l}\text { Address of the best } \\
\text { matching domain block }\end{array}$ & $A_{d}$ & $\begin{array}{c}16 \\
\text { [bits] }\end{array}$ & $18[\mathrm{bits}]$ \\
\hline
\end{tabular}

On the other hand, as described above in Figure 1, incidence of the best matching range blocks is high in the region that Euclidean distance smaller than 12 . It means that we can search similar range blocks in this region effectively, and use relative addresses to explain the position of similar range block to cut down address information.
As in the proposed method, range block size is $4 \times 4$, so we can use address within $[-3,3]$ in both $x$ and $y$-axis to represent relative position of similar range blocks.

So we set searching areas of similar range blocks to the above region. Compared with the method that searches similar range block from all of the range blocks of the image, by limiting the region of the searching to the region where the incidence of similar range block is high, the position information of the range block can be set short, and the computation of similarities between range blocks can be reduced. By the above method, $\mathrm{X}$ and $\mathrm{Y}$ coordination of the relative address are limited into 3 bits. It is far shorter than information about the best matching domain block, so the reduction in the transmitted information can be realized by the proposed method.

\section{Algorithm of the proposed method}

Algorithm of the proposed method is shown as follows.

In the encoding process, to non-flat range block $R_{1}$, the best matching range block $R_{2}$ is searched from the searching area. And then let us compare $\delta_{R_{1} R_{2}}$ as shown in (3) with threshold $T_{2}$, if $\delta_{R_{1} R_{2}}$ is smaller than $T_{2}$, we don't search the best matching domain block of range block $R_{1}$, and then transmit the position information of best matching range block $R_{2}$ to decoder; if not, the best matching domain block is searched as the current methods. In the proposed method, we set flag $F_{2}$ to each non-flat range block, and when address of best matching range block is transmitted, $F_{2}$ is set as 1 ; if address and affine information of best matching domain block are transmitted, $F_{2}$ is set as 0 .

In the decoding process, flag $F_{2}$ of range block $R_{1}$ is checked firstly; if it is 1 , the best matching range block is extracted from the location of the best matching range block, and it is used to reconstruct range block $R_{1}$. If $F_{2}$ is 0 , range block $R_{1}$ is reconstructed by the best matching domain block as the current method.

\section{Transmitted information in the proposed method}


The quantity of transmitted information in the current method $I_{c}$, and that in the proposed method $I_{p}$ is shown as follows.

$$
\begin{aligned}
I_{c}= & \left(M_{r}+S_{r}+A_{d}+\tau+F_{1}\right) \times\left(N_{1}+N_{2}\right) \\
I_{p}= & \left(M_{r}+S_{r}+A_{r}+F_{1}+F_{2}\right) \times N_{1} \\
& +\left(M_{r}+S_{r}+A_{d}+\tau+F_{1}+F_{2}\right) \times N_{2}
\end{aligned}
$$

In the above equation, $M_{r}$ is mean values of the range block, $S_{r}$ is standard deviation of the range block, $A_{d}$ is position information of the best matching domain blocks, $\tau$ is affine transformation information of the best matching domain block, $A_{r}$ is the position information of the best matching range blocks, $N_{1}$ is the number of range blocks reconstructed by the best matching range block, $N_{2}$ is the number of range blocks that are reconstructed by the best matching domain block in the proposed method. $F_{1}$ is the flag of flat/non-flat range blocks, $F_{2}$ is the flag that show the range blocks are reconstructed by the best matching range block or the best matching domain block.

Then, when differences of transmitted information $I_{\text {diff }}$ between the current and proposed methods shown in (7) is positive; we can use the proposed method to cut down the quantity of transmitted information.

$$
\begin{aligned}
I_{\text {diff }} & =I_{c}-I_{p} \\
& =\left(A_{d}+\tau\right) N_{1}-\left[\left(A_{r}+F_{2}\right) N_{1}+F_{2} N_{2}\right] \\
& >0
\end{aligned}
$$

By dividing both side by $N_{1}$ in (7),

$$
\begin{aligned}
\frac{I_{\text {diff }}}{N_{1}} & =\left(A_{d}+\tau\right)-\left[\left(A_{r}+F_{2}\right)+F_{2} n\right] \\
& >0
\end{aligned}
$$

Here,

$$
n=\frac{N_{2}}{N_{1}}
$$

$n_{b}$ which is the turning point of preferable area to the current method and the proposed method can be calculated by (10).

$$
n_{b}=\frac{\left(A_{d}+\tau\right)-\left(A_{r}+F_{2}\right)}{F_{2}}
$$

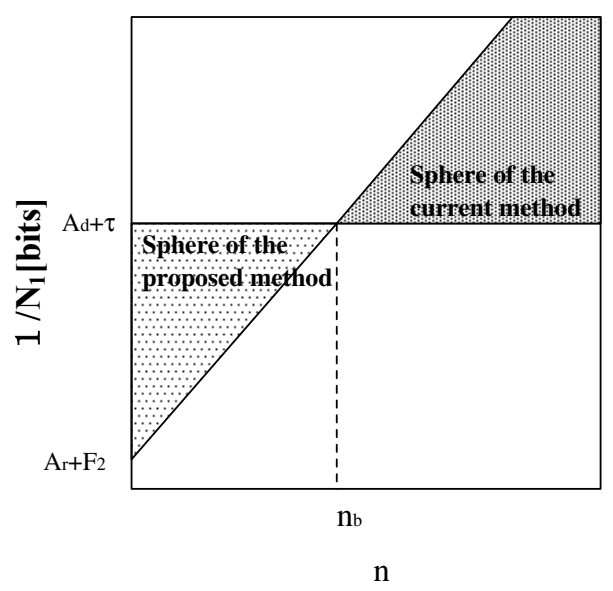

Figure 2. Preferable area for the current method and the proposed method.

Figure.2 shows a preferable area for the current method and the proposed method. When $n$ is smaller than $n_{b}$, the reduction of transmitted information can be achieved by the proposed method.

\section{Simulation}

\subsection{Influence on SNR of the reconstructed image by threshold $T_{2}$}

In the proposed method, the best matching domain blocks are not searched to the range block $R_{1}$ where $\delta_{R_{1} R_{2}}$ with the best matching range block $R_{2}$ smaller than threshold $T_{2}$, and these range blocks are reconstructed by the best matching range block. In other words, it shares the same best matching domain block with the best matching range block. Therefore, $T_{2}$ influences the reduction rate of information and SNR of the reconstructed image.

Because the bigger when $T_{2}$ gets, the more range blocks will be constructed by the best matching range block. That means that transmitted information will be cut down more, but SNR of reconstructed image will decline.

Then, we examine the number of range blocks reconstructed by the best matching range blocks $N_{1}$ and SNR of reconstructed image while $T_{2}$ is changed. Figure 3 shows the result when image Lenna is used. 
From Figure 3, we can know that when threshold $T_{2}$ gets bigger, the quantity of range blocks reconstructed by the best matching range block increases, and SNR of the reconstructed image falls. As $T_{2}$ gets bigger, range block that not similar enough with $R_{1}$ will be set as the best matching range block, finally, the error will change bigger.

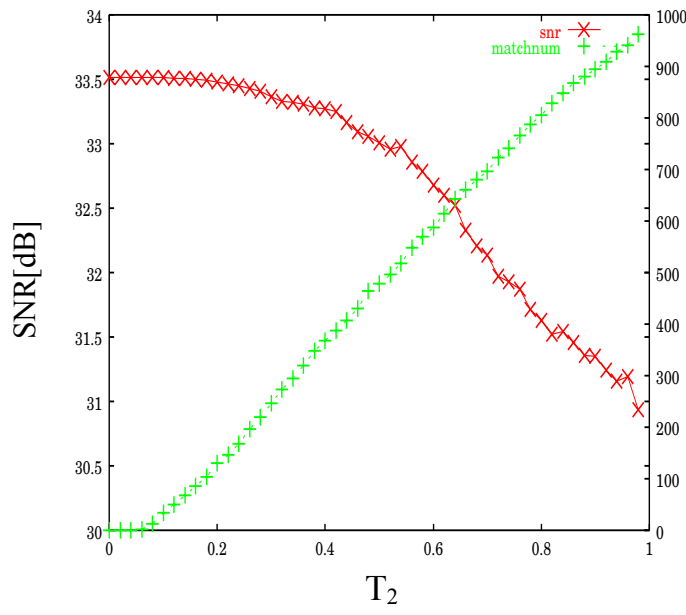

Figure 3. $T_{2}, N_{1}$ and SNR.

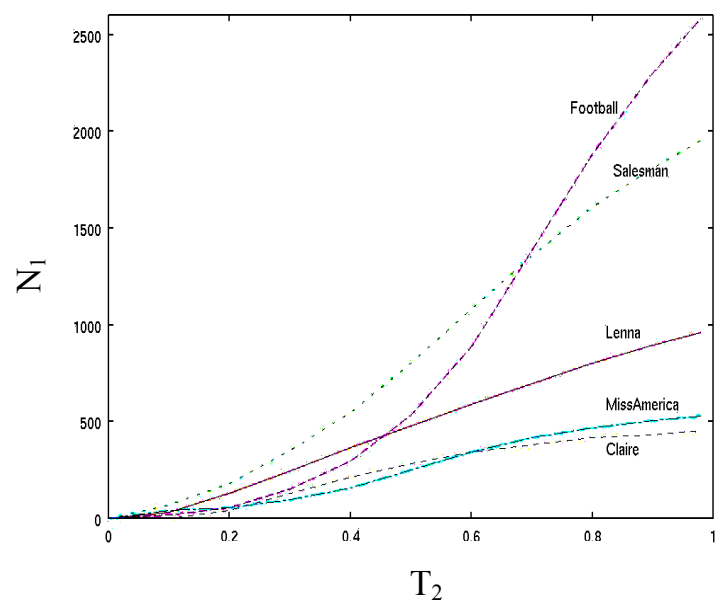

Figure 4. Relationship of $N_{1}$ and threshold $T_{2}$.

Figure 4 shows the relationship of the quantity of range blocks reconstructed by the best matching range block and threshold $T_{2}$. Figure 5 shows the relationship of the decrease of SNR and threshold $T_{2}$. From Figure 4 we can know that the quantity of range blocks reconstructed by the best matching range blocks is about $250 \sim 800$ when $T_{2}$ is 0.5 . From Figure 5, we can know that $T_{2}$ is about 0.5 when the decrease of SNR is at $0.5 \mathrm{~dB}$.

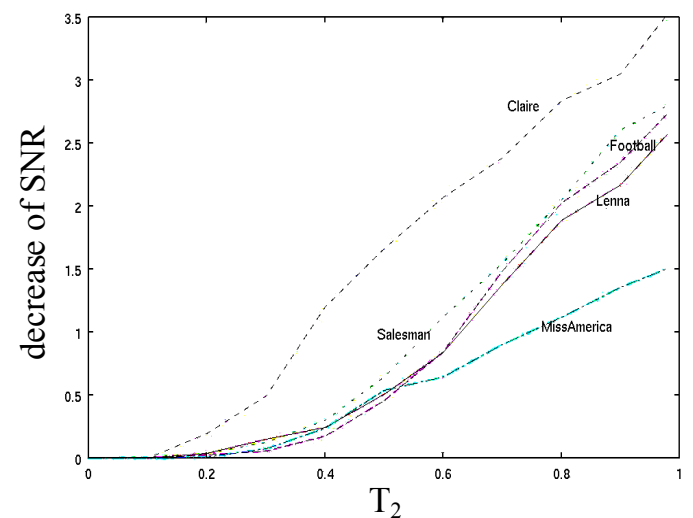

Figure 5. Relationship of $T_{2}$ and decrease of SNR.

\subsection{Reduction in the transmitted information}

In the proposed method, the quantity of transmitted information are to be shown as follows.

$$
\begin{aligned}
\mathrm{A}_{\mathrm{d}} & : 16 \mathrm{bits}(\text { Lenna), 18bits (other images) } \\
\mathrm{A}_{\mathrm{r}} & : 6 \text { bits } \\
\tau & : 3 \mathrm{bits} \\
F_{2} & : 1 \mathrm{bit}
\end{aligned}
$$

As the size of lenna is $256 \times 256$, then the address data of the best matching domain blocks $A_{d}$ is 16 bits, and the sizes of the other images are $352 \times 288$, then $A_{d}$ is 18 bits. Because $n$ should be smaller than $n_{b}$ to reduce the quantity of information by the proposed method as shown in Figure 2, (8) is as follows:

$$
\begin{aligned}
\frac{I_{\text {diff }}}{N_{1}} & =(21 \text { or } 19)-(7+n) \\
& >0
\end{aligned}
$$

Therefore, $n_{b}$ is 12 for test image Lenna, and 14 for the other test images to cut down the transmitted information. Table 2 shows $n$ and $n_{b}$ while $T_{2}$ is set at 0.5. As shown in Table 2, when threshold $T_{2}$ is 0.5, as $n$ is smaller than $n_{b}$, information reduction by the proposed method can be achieved. 
Table 2. $n$ and $n_{b}$ when $T_{2}$ is $\mathbf{0 . 5}$

\begin{tabular}{|c|c|c|c|c|}
\hline Images & $N_{2}$ & $N_{1}$ & $n$ & $n_{b}$ \\
\hline Lenna & 1692 & 478 & 3.5 & 12 \\
\hline $\begin{array}{c}\text { Salesma } \\
\mathrm{n}\end{array}$ & 3539 & 801 & 4.4 & 14 \\
\hline Claire & 687 & 284 & 2.4 & 14 \\
\hline $\begin{array}{c}\text { Miss } \\
\text { America }\end{array}$ & 905 & 252 & 3.6 & 14 \\
\hline
\end{tabular}

Finally, we simulated using the proposed method Table 3 shows the ratio of reduced information and SNR of reconstructed image.

\section{Table 3. SNR and reduced information}

\begin{tabular}{|c|c|c|}
\hline Images & SNR [dB] & Reduced information [\%] \\
\hline Lenna & 33.01 & 5.1 \\
\hline Salesman & 33.59 & 4.8 \\
\hline Claire & 40.04 & 4.2 \\
\hline $\begin{array}{c}\text { Miss } \\
\text { America }\end{array}$ & 39.19 & 3.2 \\
\hline
\end{tabular}

From Table 3, we can know that transmitted information was cut down in all images by the proposed method. Figure 6 show the original image and the reconstructed image. From these images, we can know that reconstructed image of the high image quality are obtained.

\section{Conclusion}

We have proposed a reduction of transmitted information method using similarities between range blocks. With the proposed method, address information of the best matching range block is transmitted instead of the address of the best matching domain block to cut down transmitted information.

Firstly, we examined the distances between the range blocks most similar to each other. As a result, we knew that possibility of most similar range blocks is high when distance between range blocks is small. So, the address information of the best matching range block can be set smaller than that of the best matching domain block. Simulation results showed that the proposed method has reduced the quantity of transmitted information about $5 \%$.

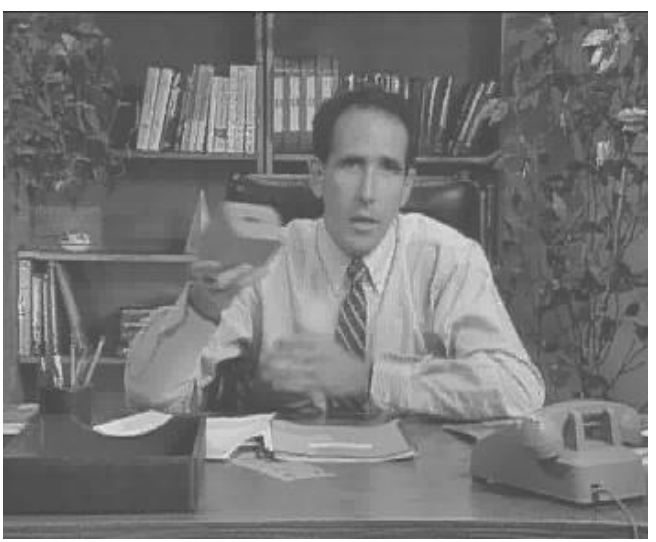

(a) Original image

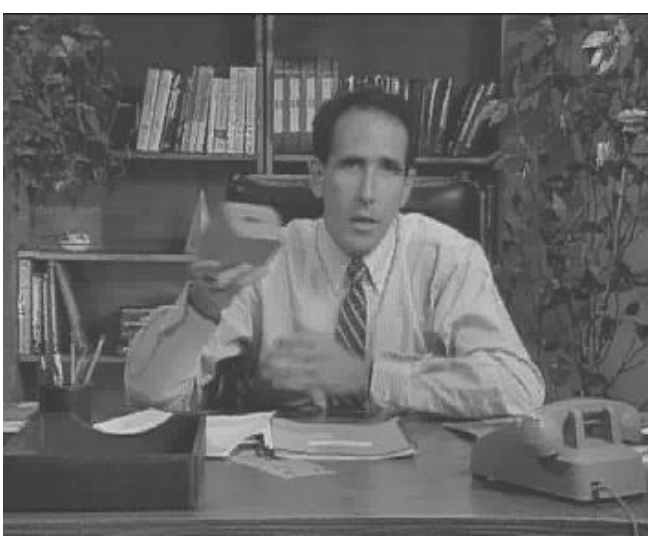

(b) Reconstructed image

Figure 6. Original image and reconstructed image (Salesman)

\section{References}

[1] A.E. Jacquin, A novel fractal-coding technique for digital images,IEEE Int. Conf. on Acoustics, Speech and Signal Processing, M8.2, Vol.4, pp.2225-2228, 1990.

[2] Xiaotong Hu, Makoto Fujimura, Yoko Maemura and Hideo Kuroda, Reduction of the number of searched Domain Blocks for Fractal Image Coding Using the Center of Gravity of the Image Block, IEICE Trans. INF.I\& SYST., Vol.E86D,No.2, pp.344-347, 2003.

[3] C.J. Wein and I.F. Blake, On the performance of fractal compression with clustering, IEEE trans. Image Processing, Vol.5, No.3, pp.522-526, 1996.

[4] D.C. Popescu and H. Yan, MR image compression using iterated function systems, Magnetic Resonance Imaging, Vol.11, No.5,pp.727-732, 1993.

[5] L.M. Beaumont, Image data compression using fractal techniques, BT Technology, Vol.9, No.4, pp.93-109, 1991.

[6] Kousuke Imamura, Makoto Fujimura and Hideo Kuroda, Fast Block Searching Method Using Pattern Index for Fractal Image Coding, Journal of the IIEE of Japan, Vol.28,No.5, pp.576-584,1999. 